\title{
Ictiofauna demersal profunda del Parque Nacional Natural Corales de Profundidad, Caribe Colombiano: primera aproximación a su biodiversidad
}

\author{
Deep demersal fish fauna of the Parque Nacional Natural Corales de Profundidad, Colombian \\ Caribbean: First approach to its biodiversity
}

Camilo B. García ${ }^{1 *}$

\begin{abstract}
Resumen
Con base en registros geo-referenciados se compone, por primera vez, una lista de especies de peces demersales profundos (>200 m) del Parque Nacional Natural Corales de Profundidad (Caribe colombiano). Se explora la completitud del inventario de especies y se propone un primer esquema de ensamblajes ícticos profundos demersales dentro del parque. Se registraron 105 especies distribuidas en 427 ocurrencias georreferenciadas. El inventario de especies está lejos de ser completo, con apenas el 32,6\% del área profunda del parque visitada, a la escala espacial del estudio. Se resalta el potencial de encontrar ensamblajes de peces dentro del área del parque con relevancia para su manejo.
\end{abstract}

Palabras claves: área protegida, completitud, inventario, peces profundos

\begin{abstract}
Georeference records of deep demersal fishes $(>200 \mathrm{~m}$ ) inside the Parque Nacional Natural Corales de Profundidad (Colombian Caribbean) were collected and a list of species is composed and presented for the first time. Completeness of this inventory is explored, and a first schema of fish assemblages is proposed. 427 records were found corresponding to 105 species. The inventory is deficient with an explored area of $32.6 \%$ of the park. The potential of fish assemblages inside the park is highlighted which may be relevant for its management.
\end{abstract}

Keywords: Protected area, completeness, inventory, deep fish fauna

\section{INTRODUCCIÓN}

Una de las métricas importantes de conservación es la diversidad de especies de una región o localidad (Capmourteres y Anand, 2016; Parsons et al., 2014). Si bien el Parque Nacional Natural Corales de Profundidad fue erigido con el fin de proteger a un grupo de fauna particular de acuerdo con su nombre (Alonso et al., 2015; Urriago et al., 2011), al obtener ese estatus los demás componentes bióticos también reciben protección. Por tanto, valorar la diversidad de los demás componentes bióticos que están dentro del perímetro del parque es importante pues representan un valor añadido a su rol de conservación. La correcta administración de la zona protegida requiere igualmente de información biológica y ecológica completa que permita acciones dirigidas dentro de la misma.

\footnotetext{
1. Departamento de Biología, Universidad Nacional de Colombia, Bogotá, Colombia.

* Autor de correspondencia: <cbgarciar@unal.edu.co>
} 
En Colombia no existe una zona protegida con foco en los peces demersales de profundidad, definidos aquí como los que ocurren a profundidades mayores de 200 m. Sin embargo, el Parque Nacional Natural Corales de Profundidad (Caribe colombiano), cuya extensión alcanza los $1234 \mathrm{~m}$ de profundidad (Morales et al., 2017), despliega su protección a este grupo de fauna, aun sin caracterizarse en el parque. Los registros de peces existentes corresponden a profundidades de $50 \mathrm{~m}$ o menos (Chasqui y González, 2019). A profundidades menores a $200 \mathrm{~m}$ se registra la presencia del pez invasor Pterois volitans (HenaoCastro et al., 2016). Por su parte, Bustos-Montes et al. (2013) registraron el pez de aguas profundas Hyporthodus nigritus a unos $200 \mathrm{~m}$ de profundidad en lo que hoy es área del parque, cuya presencia fue confirmada en este estudio (tabla 1).

Este trabajo presenta la primera lista de peces demersales profundos del Parque Nacional Natural Corales de profundidad y su análisis, en términos de su completitud. Igualmente, se presenta una propuesta preliminar de ensamblajes ícticos profundos en el área del parque.

\section{MATERIALES Y MÉTODOS}

Previamente se construyó una base de datos taxonómica y geo-referenciada (latitud, longitud y profundidad de las ocurrencias) general, para peces demersales de fondos blandos del Caribe colombiano, usando el programa ModestR (García-Roselló et al., 2013); este programa permite crear y analizar mapas de distribución de especies. Las fuentes de información de la base de datos general incluyeron los estudios de Acero et al. (2018), García (2017), García y Armenteras (2015), Global Biodiversity Information Facility (GBIF 2020), Polanco (2015) y Polanco et al. (2019). A partir de esta base de datos se seleccionaron, con la aplicación MRFinder componente de ModestR, las ocurrencias dentro del parque.

ModestR permite exportar archivos útiles para la aplicación KnowBR (Guisande y Lobo, 2019; Lobo et al., 2018) del programa RWizard (Guisande et al., 2014), diseñada para explorar la completitud de inventarios de especies en términos de unidades espaciales. Para tal efecto el área del parque fue dividida en celdas de 2 min por 2 min. Dos min de arco corresponden, aproximadamente, a $3,70 \mathrm{~km}$ en el trópico, lo que lleva a un área de celda de unos $13,7 \mathrm{~km}^{2}$. Se conservaron los argumentos por defecto para el análisis con KnowBR así: curve = "Rational" (Ratkowski, 1990) que es una de las opciones para el ajustar una función a la curva de acumulación de especies para la cual las ocurrencias operan como sucedáneas del esfuerzo de muestreo (Lobo, 2008; Lobo et al., 2018); estimator $=\mathbf{1}$, lo que significa que la curva de acumulación de especies se construyó usando la fórmula de Ugland et al. (2013); cutoff = 1, significando que si el cociente entre el número de ocurrencias ("records") y el número de especies es 1 en una unidad espacial, esta se considera no informativa y su completitud no se calcula; cutoffCompleteness $=\mathbf{0}$, indica que si el valor de completitud es menor a este valor no se calcula la completitud; cutoffSlope $=1$, significa que si la pendiente final de la curva de acumulación es mayor que este valor la completitud no se calcula. Este escenario representa la configuración menos restrictiva de estimación de la completitud.

Se usó la función SurveyQ ("survey quality") de KnowBR para representar geográficamente las celdas muestreadas bien o deficientemente. Para esto se usaron los valores por defecto de la función; para celdas bien muestreadas: pendiente $<0,02$, completitud $>90 \%$ y relación R/S ("Records"/"Species") > 15 . Para celdas deficientemente muestreadas: pendiente $>0,3$, completitud $<50 \%$ y relación $\mathrm{R} / \mathrm{S}<3$.

Con la aplicación MRGrapher de ModestR se exploró la existencia de agrupamientos distinguibles (ensamblajes) de especies con base en el porcentaje de solapamiento entre distribuciones de todos los posibles pares de especies, definiéndose cada distribución por su extensión de ocurrencia o "extent of ocurrence", EOO (IUCN, 2012). Con la matriz de \% de solapamientos, la aplicación MRGrapher permite buscar recurrencia de especies mediante el procedimiento de agrupamiento de $k$-medias optimizado de manera que la suma de las distancias entre los centroides de los grupos formados sea la máxima. 
Tabla 1. Lista de las especies profundas $(>200 \mathrm{~m})$ y su número de ocurrencias encontradas dentro del área del Parque Nacional Natural Corales de Profundidad

\begin{tabular}{|c|c|c|c|c|c|}
\hline Clase & Orden & Familia & Genero & Especie & Ocurrencias \\
\hline Actinopterygii & Anguilliformes & Colocongridae & Coloconger & Coloconger meadi & 5 \\
\hline Actinopterygii & Anguilliformes & Congridae & Bathycongrus & Bathycongrus bullisi & 2 \\
\hline Actinopterygii & Anguilliformes & Congridae & Pseudophichthys & Pseudophichthys splendens & 2 \\
\hline Actinopterygii & Anguilliformes & Congridae & Xenomystax & Xenomystax congroides & 4 \\
\hline Actinopterygii & Aulopiformes & Chlorophthalmidae & Chlorophthalmus & Chlorophthalmus agassizi & 20 \\
\hline Actinopterygii & Aulopiformes & Chlorophthalmidae & Parasudis & Parasudis truculenta & 3 \\
\hline Actinopterygii & Aulopiformes & Ipnopidae & Bathypterois & Bathypterois bigelowi & 6 \\
\hline Actinopterygii & Aulopiformes & Synodontidae & Saurida & Saurida brasiliensis & 2 \\
\hline Actinopterygii & Beryciformes & Trachichthyidae & Hoplostethus & Hoplostethus occidentalis & 4 \\
\hline Actinopterygii & Gadiformes & Bregmacerotidae & Bregmaceros & Bregmaceros atlanticus & 3 \\
\hline Actinopterygii & Gadiformes & Macrouridae & Bathygadus & Bathygadus macrops & 2 \\
\hline Actinopterygii & Gadiformes & Macrouridae & Coelorinchus & Coelorinchus caelorhincus & 10 \\
\hline Actinopterygii & Gadiformes & Macrouridae & Coelorinchus & Coelorinchus caribbaeus & 2 \\
\hline Actinopterygii & Gadiformes & Macrouridae & Gadomus & Gadomus arcuatus & 2 \\
\hline Actinopterygii & Gadiformes & Macrouridae & Hymenocephalus & Hymenocephalus billsam & 2 \\
\hline Actinopterygii & Gadiformes & Macrouridae & Hymenocephalus & Hymenocephalus italicus & 7 \\
\hline Actinopterygii & Gadiformes & Macrouridae & Malacocephalus & Malacocephalus laevis & 2 \\
\hline Actinopterygii & Gadiformes & Macrouridae & Malacocephalus & Malacocephalus occidentalis & 7 \\
\hline Actinopterygii & Gadiformes & Macrouridae & Nezumia & Nezumia aequalis & 14 \\
\hline Actinopterygii & Gadiformes & Macrouridae & Ventrifossa & Ventrifossa macropogon & 2 \\
\hline Actinopterygii & Gadiformes & Merlucciidae & Steindachneria & Steindachneria argentea & 7 \\
\hline Actinopterygii & Gadiformes & Moridae & Gadella & Gadella imberbis & 2 \\
\hline Actinopterygii & Gadiformes & Moridae & Laemonema & Laemonema goodebeanorum & 7 \\
\hline Actinopterygii & Gadiformes & Moridae & Physiculus & Physiculus fulvus & 2 \\
\hline Actinopterygii & Lophiiformes & Chaunacidae & Chaunax & Chaunax pictus & 5 \\
\hline Actinopterygii & Lophiiformes & Chaunacidae & Chaunax & Chaunax suttkusi & 14 \\
\hline Actinopterygii & Lophiiformes & Lophiidae & Lophiodes & Lophiodes monodi & 2 \\
\hline Actinopterygii & Lophiiformes & Ogcocephalidae & Dibranchus & Dibranchus atlanticus & 14 \\
\hline Actinopterygii & Lophiiformes & Ogcocephalidae & Halieutichthys & Halieutichthys aculeatus & 6 \\
\hline Actinopterygii & Lophiiformes & Ogcocephalidae & Malthopsis & Malthopsis gnoma & 2 \\
\hline Actinopterygii & Lophiiformes & Ogcocephalidae & Ogcocephalus & Ogcocephalus declivirostris & 2 \\
\hline Actinopterygii & Myctophiformes & Myctophidae & Dasyscopelus & Dasyscopelus selenops & 2 \\
\hline Actinopterygii & Myctophiformes & Myctophidae & Diaphus & Diaphus effulgens & 2 \\
\hline Actinopterygii & Myctophiformes & Myctophidae & Diaphus & Diaphus garmani & 2 \\
\hline Actinopterygii & Myctophiformes & Myctophidae & Diaphus & Diaphus lucidus & 2 \\
\hline Actinopterygii & Myctophiformes & Neoscopelidae & Neoscopelus & Neoscopelus macrolepidotus & 6 \\
\hline Actinopterygii & Myctophiformes & Neoscopelidae & Neoscopelus & Neoscopelus microchir & 7 \\
\hline Actinopterygii & Notacanthiformes & Halosauridae & Halosaurus & Halosaurus guentheri & 2 \\
\hline Actinopterygii & Notacanthiformes & Halosauridae & Halosaurus & Halosaurus ovenii & 5 \\
\hline Actinopterygii & Notacanthiformes & Notacanthidae & Notacanthus & Notacanthus chemnitzii & 2 \\
\hline Actinopterygii & Ophidiiformes & Bythitidae & Calamopteryx & Calamopteryx robinsorum & 2 \\
\hline Actinopterygii & Ophidiiformes & Bythitidae & Diplacanthopoma & Diplacanthopoma brachysoma & 6 \\
\hline Actinopterygii & Ophidiiformes & Ophidiidae & Benthocometes & Benthocometes robustus & 2 \\
\hline Actinopterygii & Ophidiiformes & Ophidiidae & Monomitopus & Monomitopus agassizii & 2 \\
\hline Actinopterygii & Ophidiiformes & Ophidiidae & Neobythites & Neobythites gilli & 9 \\
\hline Actinopterygii & Ophidiiformes & Ophidiidae & Neobythites & Neobythites marginatus & 9 \\
\hline Actinopterygii & Osmeriformes & Argentinidae & Argentina & Argentina striata & 5 \\
\hline Actinopterygii & Perciformes & Acropomatidae & Caraibops & Caraibops trispinosus & 2 \\
\hline Actinopterygii & Perciformes & Acropomatidae & Synagrops & Synagrops bellus & 8 \\
\hline Actinopterygii & Perciformes & Acropomatidae & Verilus & Verilus atlanticus & 2 \\
\hline Actinopterygii & Perciformes & Acropomatidae & Verilus & Verilus pseudomicrolepis & 2 \\
\hline Actinopterygii & Perciformes & Bathyclupeidae & Bathyclupea & Bathyclupea argentea & 2 \\
\hline Actinopterygii & Perciformes & Bathyclupeidae & Bathyclupea & Bathyclupea schroederi & 6 \\
\hline
\end{tabular}




\begin{tabular}{|c|c|c|c|c|c|}
\hline Clase & Orden & Familia & Genero & Especie & Ocurrencias \\
\hline Actinopterygii & Perciformes & Caproidae & Antigonia & Antigonia capros & 4 \\
\hline Actinopterygii & Perciformes & Caproidae & Antigonia & Antigonia combatia & 11 \\
\hline Actinopterygii & Perciformes & Carangidae & Decapterus & Decapterus tabl & 2 \\
\hline Actinopterygii & Perciformes & Carangidae & Selar & Selar crumenophthalmus & 2 \\
\hline Actinopterygii & Perciformes & Epigonidae & Epigonus & Epigonus macrops & 1 \\
\hline Actinopterygii & Perciformes & Epigonidae & Epigonus & Epigonus occidentalis & 6 \\
\hline Actinopterygii & Perciformes & Epigonidae & Epigonus & Epigonus pandionis & 4 \\
\hline Actinopterygii & Perciformes & Lutjanidae & Pristipomoides & Pristipomoides macrophthalmus & 2 \\
\hline Actinopterygii & Perciformes & Opistognathidae & Lonchopisthus & Lonchopisthus lemur & 4 \\
\hline Actinopterygii & Perciformes & Percophidae & Bembrops & Bembrops anatirostris & 2 \\
\hline Actinopterygii & Perciformes & Percophidae & Bembrops & Bembrops gobioides & 1 \\
\hline Actinopterygii & Perciformes & Percophidae & Bembrops & Bembrops ocellatus & 5 \\
\hline Actinopterygii & Perciformes & Percophidae & Bembrops & Bembrops quadrisella & 1 \\
\hline Actinopterygii & Perciformes & Serranidae & Baldwinella & Baldwinella aureorubens & 3 \\
\hline Actinopterygii & Perciformes & Serranidae & Bathyanthias & Bathyanthias cubensis & 2 \\
\hline Actinopterygii & Perciformes & Serranidae & Hyporthodus & Hyporthodus nigritus & 1 \\
\hline Actinopterygii & Perciformes & Symphysanodontidae & Symphysanodon & Symphysanodon berryi & 3 \\
\hline Actinopterygii & Perciformes & Trichiuridae & Benthodesmus & Benthodesmus simonyi & 2 \\
\hline Actinopterygii & Perciformes & Trichiuridae & Benthodesmus & Benthodesmus tenuis & 3 \\
\hline Actinopterygii & Pleuronectiformes & Bothidae & Monolene & Monolene atrimana & 2 \\
\hline Actinopterygii & Pleuronectiformes & Bothidae & Monolene & Monolene megalepis & 2 \\
\hline Actinopterygii & Pleuronectiformes & Paralichthyidae & Ancylopsetta & Ancylopsetta cycloidea & 2 \\
\hline Actinopterygii & Pleuronectiformes & Pleuronectidae & Poecilopsetta & Poecilopsetta beanii & 1 \\
\hline Actinopterygii & Pleuronectiformes & Pleuronectidae & Poecilopsetta & Poecilopsetta inermis & 5 \\
\hline Actinopterygii & Polymixiiformes & Polymixiidae & Polymixia & Polymixia lowei & 7 \\
\hline Actinopterygii & Scorpaeniformes & Peristediidae & Peristedion & Peristedion ecuadorense & 4 \\
\hline Actinopterygii & Scorpaeniformes & Peristediidae & Peristedion & Peristedion gracile & 2 \\
\hline Actinopterygii & Scorpaeniformes & Peristediidae & Peristedion & Peristedion greyae & 1 \\
\hline Actinopterygii & Scorpaeniformes & Peristediidae & Peristedion & Peristedion longispatha & 6 \\
\hline Actinopterygii & Scorpaeniformes & Peristediidae & Peristedion & Peristedion truncatum & 2 \\
\hline Actinopterygii & Scorpaeniformes & Scorpaenidae & Neomerinthe & Neomerinthe beanorum & 2 \\
\hline Actinopterygii & Scorpaeniformes & Scorpaenidae & Pontinus & Pontinus nematophthalmus & 2 \\
\hline Actinopterygii & Scorpaeniformes & Setarchidae & Setarches & Setarches guentheri & 12 \\
\hline Actinopterygii & Stomiiformes & Phosichthyidae & Polymetme & Polymetme corythaeola & 2 \\
\hline Actinopterygii & Stomiiformes & Phosichthyidae & Polymetme & Polymetme thaeocoryla & 2 \\
\hline Actinopterygii & Stomiiformes & Sternoptychidae & Argyripnus & Argyripnus atlanticus & 2 \\
\hline Actinopterygii & Stomiiformes & Sternoptychidae & Argyropelecus & Argyropelecus aculeatus & 5 \\
\hline Actinopterygii & Stomiiformes & Sternoptychidae & Argyropelecus & Argyropelecus gigas & 2 \\
\hline Actinopterygii & Stomiiformes & Sternoptychidae & Maurolicus & Maurolicus muelleri & 2 \\
\hline Actinopterygii & Stomiiformes & Sternoptychidae & Polyipnus & Polyipnus asteroides & 4 \\
\hline Actinopterygii & Stomiiformes & Sternoptychidae & Polyipnus & Polyipnus laternatus & 2 \\
\hline Actinopterygii & Stomiiformes & Stomiidae & Chauliodus & Chauliodus sloani & 14 \\
\hline Actinopterygii & Tetraodontiformes & Triacanthodidae & Parahollardia & Parahollardia schmidti & 2 \\
\hline Actinopterygii & Zeiformes & Grammicolepididae & Xenolepidichthys & Xenolepidichthys dalgleishi & 2 \\
\hline Actinopterygii & Zeiformes & Parazenidae & Cyttopsis & Cyttopsis rosea & 10 \\
\hline Actinopterygii & Zeiformes & Zenionidae & Zenion & Zenion hololepis & 12 \\
\hline Elasmobranchii & Carcharhiniformes & Scyliorhinidae & Scyliorhinus & Scyliorhinus boa & 2 \\
\hline Elasmobranchii & Rajiformes & Rajidae & Breviraja & Breviraja spinosa & 2 \\
\hline Elasmobranchii & Rajiformes & Rajidae & Dipturus & Dipturus bullisi & 2 \\
\hline Elasmobranchii & Rajiformes & Rajidae & Gurgesiella & Gurgesiella atlantica & 2 \\
\hline Holocephali & Chimaeriformes & Chimaeridae & Hydrolagus & Hydrolagus alberti & 2 \\
\hline Myxini & Myxiniformes & Myxinidae & Myxine & Myxine mccoskeri & 2 \\
\hline
\end{tabular}




\section{RESULTADOS}

La figura 1 muestra la posición del parque en el Caribe colombiano y las ocurrencias de peces profundos (>200 m) dentro de este. Nótese que varias ocurrencias, si bien cerca de la isobata de $200 \mathrm{~m}$ (batimetría de Natural Earth, 2020), resultan por encima de la misma, posiblemente por inexactitudes de medición en las fuentes. En todo caso, la cercanía es tal que esas ocurrencias siguen perteneciendo al ambiente profundo. En total se encontraron 105 especies repartidas en 427 ocurrencias en el área del parque (tabla 1). La especie más común fue Chloropthalmus agassizi con 20 ocurrencias; 64 especies presentaron tres o menos ocurrencias (tabla 1), lo que indica un muestreo muy parcial hasta el momento, pero también concuerda con la distribución observada en bases de datos grandes: pocas especies comunes y una larga lista de especies raras.

Con al menos una ocurrencia se contaron 15 celdas de 2 min por 2 min. Para diez de estas se calculó la completitud; no así para las restantes cinco pues su cociente $\mathrm{R} / \mathrm{S}$ fue 1 . La figura 2 muestra las ocurrencias por celda y la figura 3 muestra la riqueza observada por celda. Las celdas han sido irregularmente muestreadas con 1 a 115 ocurrencias y 1 a 44 especies por celda (figuras 2 y 3 ).

La completitud en las celdas informativas osciló entre el $28,3 \%$ y el $69,7 \%$ con una media de $47,8 \% \pm 26,7$ DS (figura 4). De las diez celdas informativas, no se observó ninguna de alta calidad de muestreo, cuatro resultaron de calidad media y seis fueron de calidad de muestreo deficiente (figura 5).

Considerando que el área del parque debajo de los $200 \mathrm{~m}$ es de aproximadamente $630 \mathrm{~km}^{2}$ y que la suma del área de las 15 celdas con al menos una ocurrencia es de $205,5 \mathrm{~km}^{2}$, se exploró, si bien en forma irregular, un $32,6 \%$ del área profunda del parque, a la escala espacial presente.

La figura 6 muestra el agrupamiento de las especies mediante $k$-medias. Las 105 especies detectadas en el área del parque se distribuyen en 11 grupos de tamaño variable, desde un grupo formado por una sola especie (Peristedion greyae) hasta grupos con más de 20 especies (figura 6).

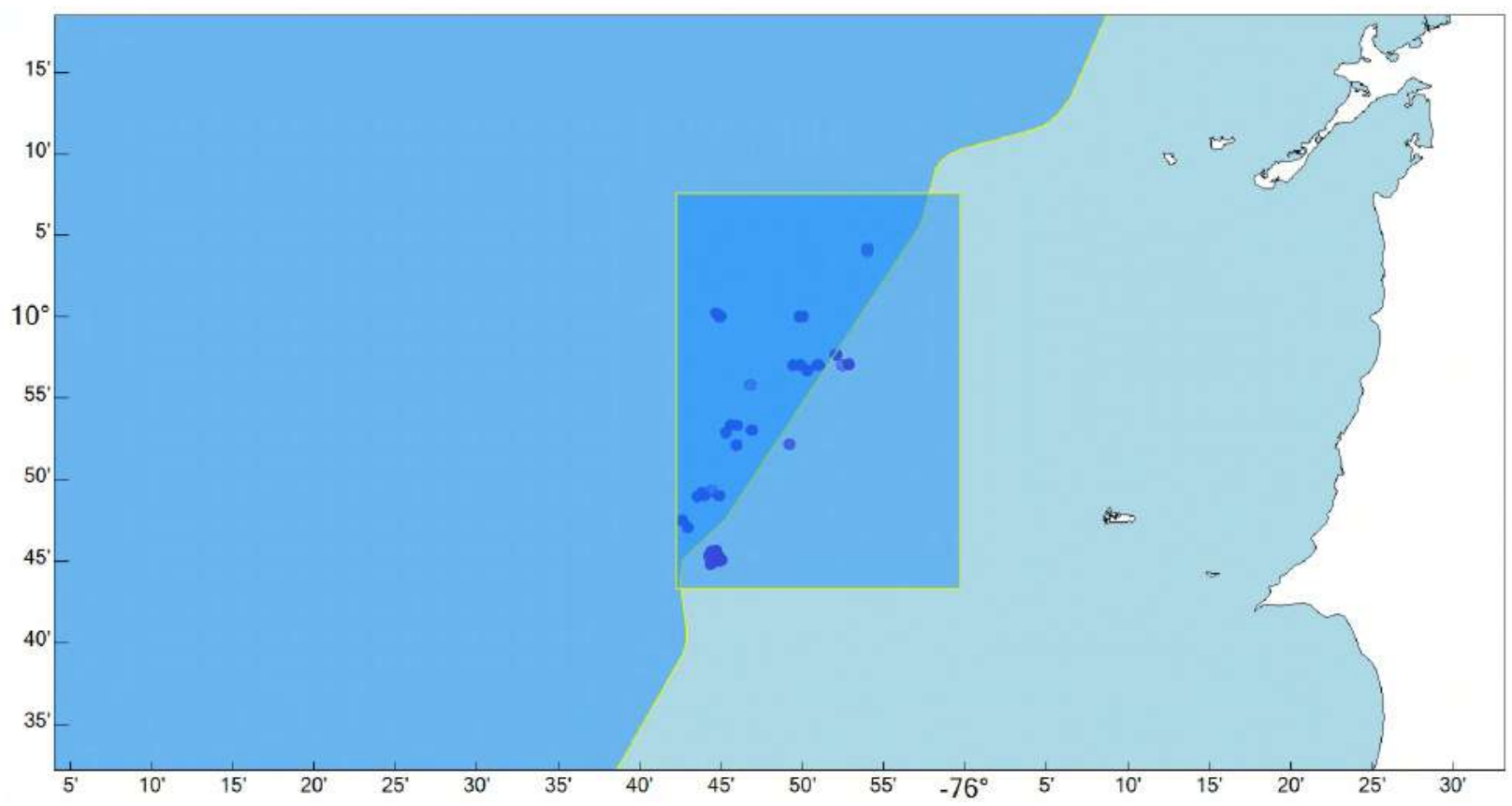

Figura 1. Mapa mostrando las ocurrencias de las especies profundas ( $>200 \mathrm{~m}$ ) encontradas dentro del Parque Nacional Natural Corales de Profundidad. El rectángulo representa el perímetro del parque. 


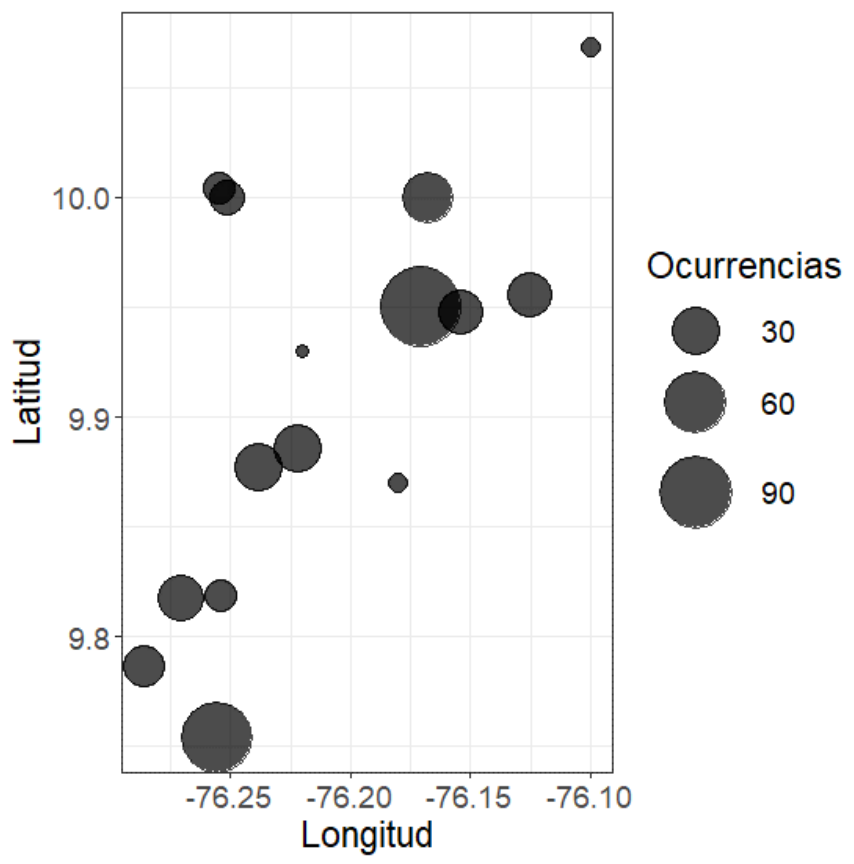

Figura 2. Distribución espacial de las ocurrencias de peces profundos $(>200 \mathrm{~m}$ ) dentro del Parque Nacional Natural Corales de Profundidad en celdas de 2 min por 2 min.

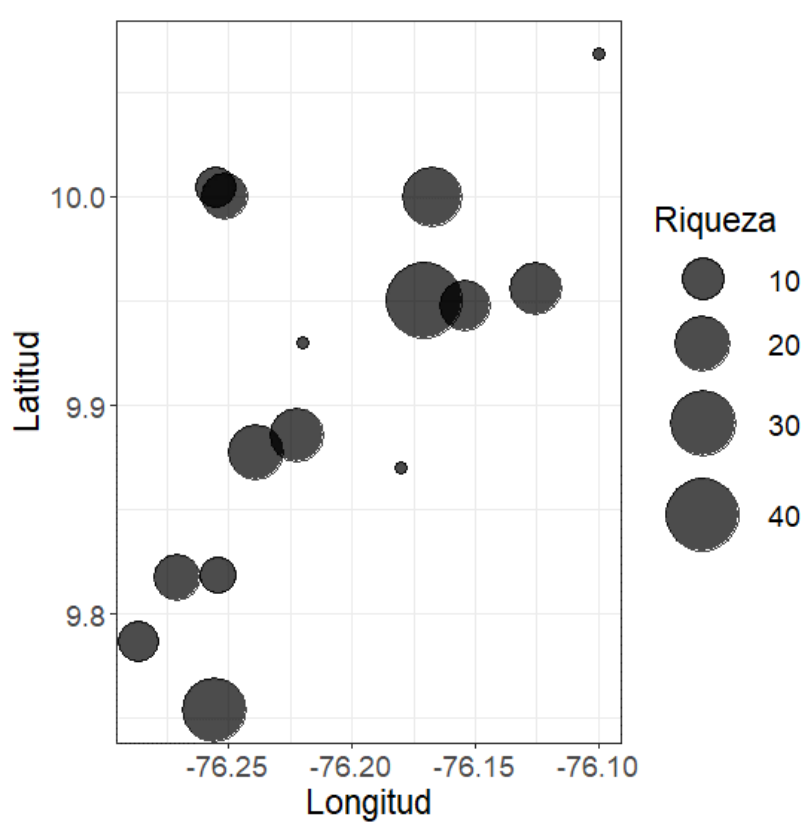

Figura 3. Distribución espacial de la riqueza de peces profundos $(>200 \mathrm{~m})$ dentro del Parque Nacional Natural Corales del Profundidad en celdas de 2 min por 2 min.

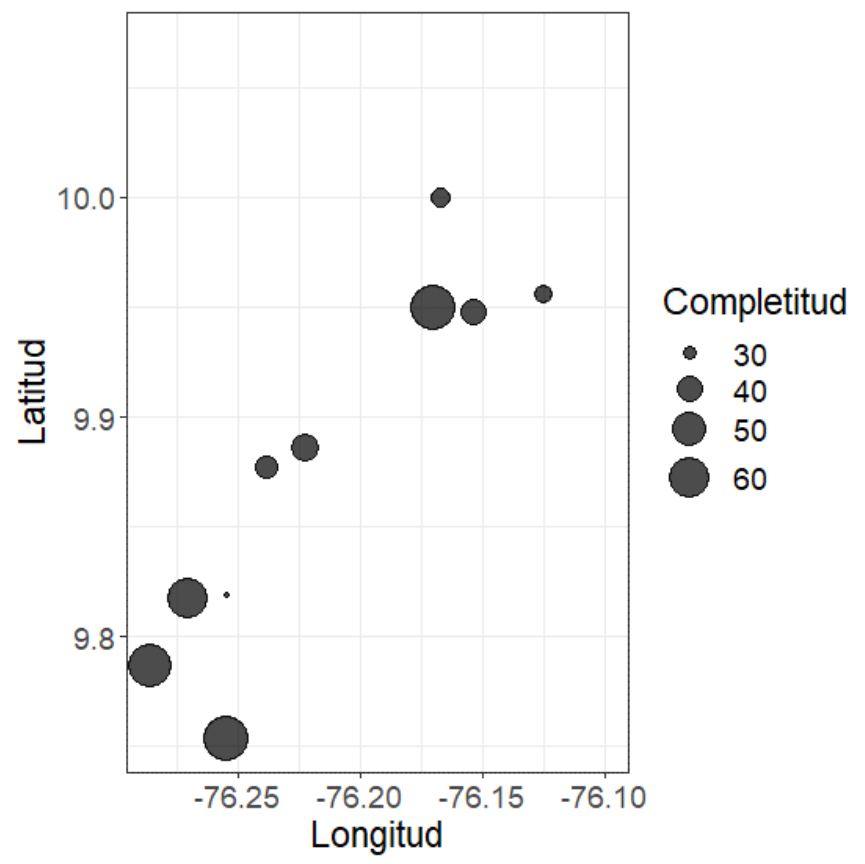

Figura 4. Distribución espacial de la completitud de muestreo de peces profundos $(>200 \mathrm{~m}$ ) dentro del Parque Nacional Natural Corales de Profundidad en celdas de 2 min por 2 min.

\section{DISCUSIÓN}

Las 105 especies de peces demersales de fondos blandos detectadas constituyen el límite inferior de la riqueza de especies ícticas profundas del parque. Es claro que hay un campo amplio y, por tanto, necesidad de futuras campañas de muestreo considerando los hallazgos globales de este estudio: solo un poco más del $30 \%$ del área bajo los $200 \mathrm{~m}$ de profundidad ha sido visitada alguna vez; la completitud promedio de los inventarios a escala de celdas de 2 min por 2 min es menor al $50 \%$; y la irregularidad del muestreo, con 5 de las 15 celdas con al menos una ocurrencia, resultando no informativas. En consecuencia, es de esperarse que próximas campañas aumenten el inventario de especies y aumenten el número de ocurrencias, es decir incremento, en zonas ocupadas del parque, de las especies ya detectadas.

Usando tanto la misma aproximación metodológica como los criterios de selección incluidos en el presente trabajo, García y Gamboa (2020) registraron 362 especies de peces profundos para el Caribe colombia- 


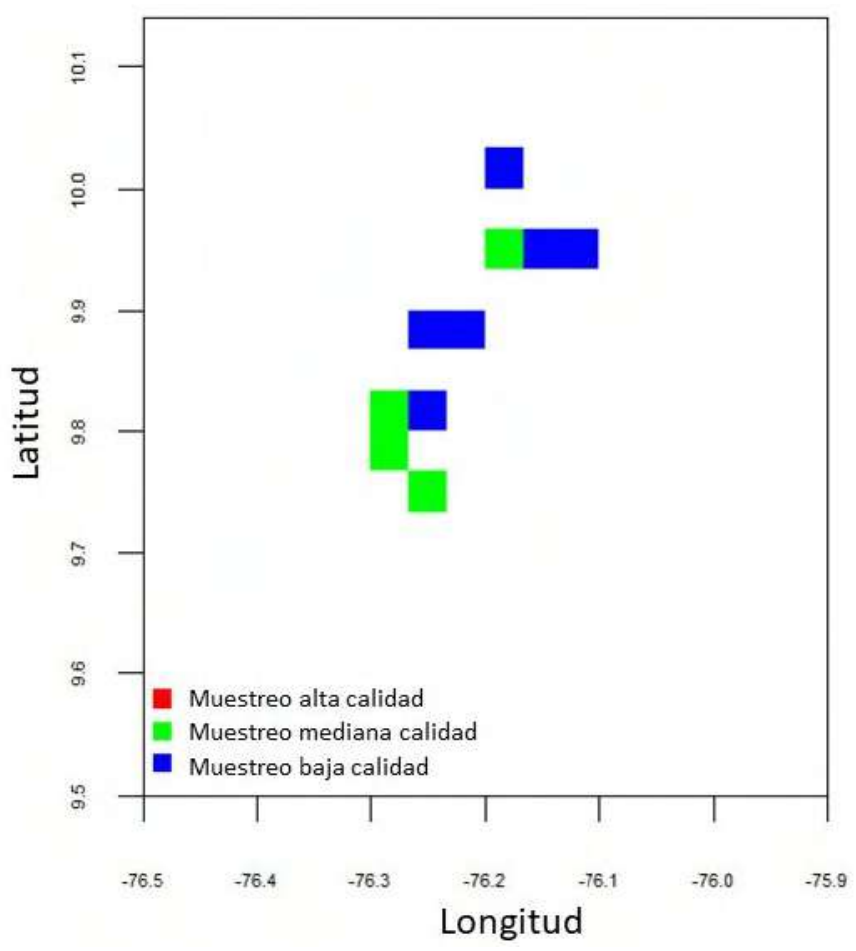

Figura 5. Distribución espacial de la calidad de muestreo de los peces profundos ( $>200 \mathrm{~m}$ ) en celdas de 2 min por 2 min que resultaron informativas dentro del Parque Nacional Natural Corales de Profundidad.

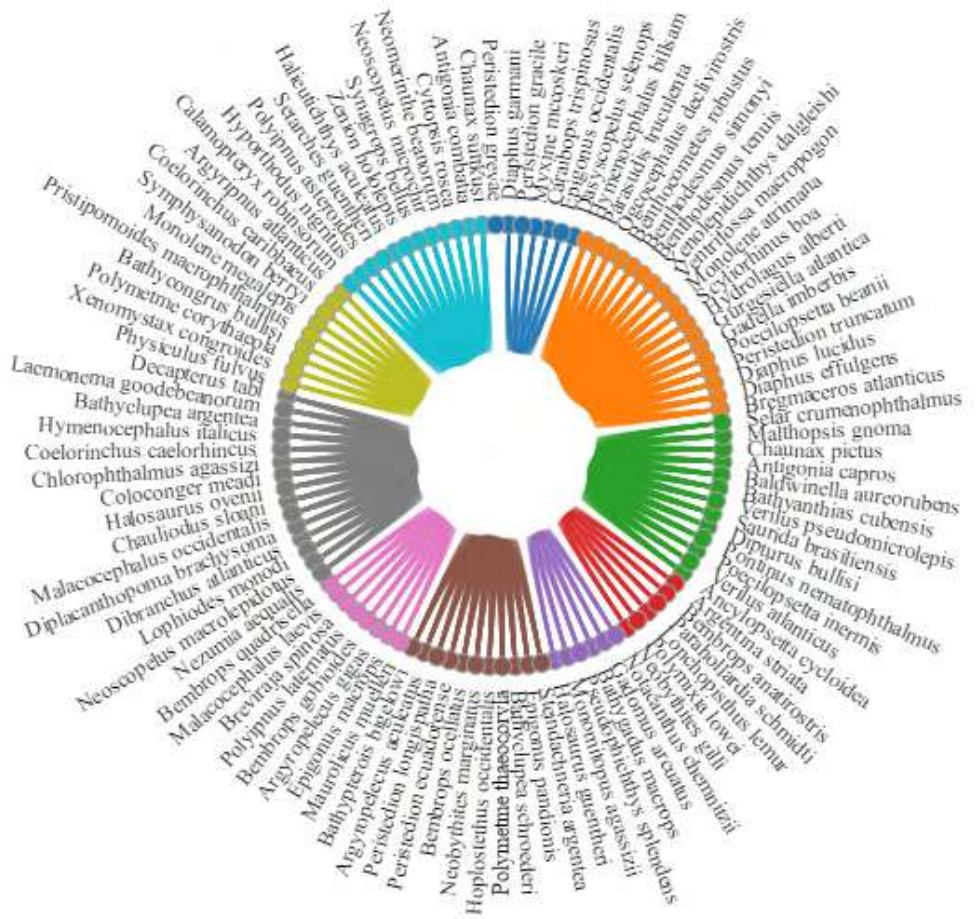

Figura 6. Propuesta de ensamblajes de peces profundos $(>200 \mathrm{~m}$ ) dentro del perímetro del Parque Nacional Natural Corales de Profundidad mediante la técnica de $k$-medias. 
no, igualmente como límite inferior de diversidad. Por tanto, de mantenerse las proporciones con futuras exploraciones, aproximadamente el $30 \%$ de la diversidad íctica demersal profunda del Caribe colombiano estaría bajo protección nominal en el Parque Nacional Natural Corales del Rosario. Con ello se pone de relieve la relevancia del Parque más allá de la protección de los corales de profundidad.

La propuesta de ensamblajes, encerrada en la figura 6 , debe verse como muy preliminar en razón a las señaladas deficiencias del inventario por lo cual no se discute más allá. Se demuestra, sin embargo, el potencial de distinguir ensamblajes de peces profundos demersales de fondos blandos dentro del perímetro del parque, lo cual representa un insumo importante en su administración.

Si bien hay espacio para el escepticismo en cuanto a la protección efectiva de los valores biológicos en los parques nacionales naturales del país, incluyendo las áreas marinas protegidas (Ramírez, 2016), con este estudio es claro que, inadvertidamente y al menos potencialmente, el efecto de conservación del parque se extiende a un grupo de fauna muy importante como son los peces demersales profundos cuya alta diversidad queda demostrada en esta investigación.

\section{CONFLICTO DE INTERESES}

El autor manifiesta no presentar conflicto de intereses.

\section{REFERENCES}

Acero, A., Polo-Silva, C.J., León, G., \& Puentes, V. (2018). First report of a sleeper shark (Somniosus sp.) in the southern Colombian Caribbean. Journal of Applied Ichthyology, 34(4), 981983. DOI:10.1111/jai.13712

Alonso, D., Vides, M., Cedeño, C., Marrugo, M., Henao, A., Sánchez, J.A., Dueñas, L., Andrade, J.C., González, F., \& Gómez, M. (2015). Parque Nacional Natural Corales de Profundidad: descripción de comunidades coralinas y fauna asociada. Serie de Publicaciones Generales del Invemar, 88, 120. http://www.invemar.org.co/documents/ 10182/14479/corales_de_profundidad.pdf

Bustos-Montes, D., Viaña-Tous, J., Acero, A., Pardo, E.R., Garrido, M., Rueda, M., \& Alonso, D. 2013. Registro de un mero negro adulto, Hyporthodus nigritus (Perciformes: Epinephelidae), en un arrecife profundo del Caribe co- lombiano. Boletín de Investigaciones Marinas y Costeras, 42(2), 413419. DOI:10.25268/bimc.invemar.2013.42.2.45

Capmourteres, V., \& Anand, M. (2016). "Conservation value": a review of the concept and its quantification. Ecosphere, 7(10), Article e01476. DOI:10.1002/ecs2.1476

Chasqui, L.H., \& Gonzáles, J.D. (2019). Peces registrados en ambientes mesofóticos de Bajo Frijol, la porción más somera del Parque Nacional Natural Corales de Profundidad, usando buceo técnico CCR. Boletín de Investigaciones Marinas y Costeras, 48(1), 89-101. DOI:10.25268/bimc.invemar.2019.48.1.759

García, C.B., \& Armenteras, D. (2015). Atlas de la ictiofauna demersal de fondos blandos del Caribe continental colombiano: Aproximación a su biodiversidad. Universidad Nacional de Colombia, Publicaciones Facultad de Ciencias. https://repositorio.unal.edu.co/handle/ unal $/ 78654$ ?show $=$ full

García, C.B. (2017). Presence and distribution of Chimaeriformes in the Colombian Caribbean Sea. Pan-American Journal of Aquatic Sciences, 12(1), 8588. https:// panamjas.org/pdf_artigos/PANAMJAS_12(1)_85-88.pdf

García, C.B., \& Gamboa, J.M. (2020). Assessment of deep demersal fish fauna diversity of the Colombian Caribbean Sea. Marine and Fishery Sciences, 33(2), 227246. DOI:10.47193/mafis.3322020301106

García-Roselló, E., Guisande, C., Gonzáles-Dacosta, J., Heine, J., Pelayo-Villamil, P., Manjarrés-Hernández, A., Vaamonde, A., \& Granado-Lorencio, C. (2013). ModestR: A software tool for managing and analyzing species distribution map databases. Ecography, 36, 12021207. DOI:10.1111/j.1600-0587.2013.00374.x

GBIF. (2020). Global Information Facility. Recuperado en junio, 2020. https://www.gbif.org/

Guisande, C., Heine, J., González-DaCosta, J., \& GarcíaRoselló, E. (2014). RWizard Software. http://www.ipez. es/RWizard. University of Vigo. Vigo, Spain.

Guisande, C., \& Lobo, J.M. (2019). Discriminating well surveyed spatial units from exhaustive biodiversity databases. $\mathrm{R}$ package version. 2.0. https://cran.r-project.org/web/ packages/KnowBR

Henao-Castro, A., Marrugo, M., \& Molina, M.P. (2016). Invasión del pez león (Pterois volitans) en ecosistemas profundos del Caribe colombiano: Parque Nacional Natural Corales de Profundidad. La Timonera, 25, 61-63.

IUCN (SSC). (2012). IUCN Red List Categories and Criteria: Version 3.1. ( $2^{\text {nd }}$ Ed.). Gland, Switzerland and Cambridge. https://www.iucn.org/content/iucn-red-listcategories-and-criteria-version-31-second- edition

Lobo, J.M. (2008). Database records as a surrogate for sampling effort provide higher species richness estimations. Biodiversity and Conservation, 17, 873881. DOI:10.1007/s10531-008-9333-4

Lobo, J.M., Hortal, J., Yelab, J.L., Millán, A., SánchezFernández, D., García-Roselló, E., González-Dacosta, J., Heinee, J., González-Vilas, L., \& Guisande, C. (2018). KnowBR: An application to map the geographical variation of survey effort and identify well-surveyed areas from biodiversity databases. Ecological Indicators, 91, 241248. DOI:10.1016/j.ecolind.2018.03.077

Morales, D., Rocha, V., \& Posada, B. (2017). Geomorfología 
de los fondos submarinos del Parque Nacional Natural Corales de Profundidad, mar Caribe colombiano. Boletín de Investigaciones Marinas y Costeras, 46(2), 7390. DOI:10.25268/bimc.invemar.2017.46.2.727

Natural Earth (2020). Free vector and raster map data at 1:10m, 1:50m, and 1:110m scales. Recuperado de https: //www.naturalearthdata.com/

Parsons, E.C.M., Favaro, B., Alonso-Aguirre, A., Bauer, A.L., Blight, L.K., Cigliano, J.A., Coleman, M.A., Côté, I.M., Draheim, M., Fletcher, S., Foley, M.M., Jefferson, R., Jones, M.C., Kelaher, B.P., Lundquist, C.J., Mccarthy, J.B., Nelson, A., Patterson, K., Walsh, L., Wright, A.J., \& Sutherland, W.J. (2014). Seventy-one important questions for the conservation of marine biodiversity. Conservation Biology, 28(5), 12061214. DOI:10.1111/cobi.12303

Polanco, A. (2015). Dynamics of the continental slope demersal fish community in the Colombian Caribbean Deep-sea research in the Caribbean. [Doctoral dissertation, Justus-Liebig University Giessen - Universidad Nacional de Colombia]. https://repositorio.unal.edu.co/ handle/unal/53827

Polanco, A., Dueñas, L.F., León, J., \& Puentes, V. (2019). New records and update on the geographic distribution of the Bony-eared Assfish, Acanthonus armatus Günther, 1878 (Ophidiidae, Neobythitinae), in the Caribbean region. Check List, 15(5), 767772. DOI:10.15560/15.5.767

Ramírez, L.F. (2016). Marine protected areas in Colombia: Advances in conservation and barriers for effective governance. Ocean \& Coastal Management, 125, 4962. DOI:10.1016/j.ocecoaman.2016.03.005

Ratkowski, D.A., \& Giles, D. (1990). Handbook of nonlinear regression models. Marcel Dekker.

Ugland, K.I., Gray, J.S., \& Ellingsen, K.E. (2003). The species-accumulation curve and estimation of species richness. Journal of Animal Ecology, 72, 888897. DOI:10.1046/j.1365-2656.2003.00748.x

Urriago, J.D., Santodomingo, N., \& Reyes, J. (2011). Formaciones coralinas de profundidad: criterios biológicos para la conformación de áreas marinas protegidas del margen continental (100-300 m) en el Caribe colombiano. Boletín Instituto de Investigaciones Marinas y Costeras, 40(1), 89-113. 